\title{
Comparative analysis of carrying capacity indices for the central Aegean islands
}

\author{
D. G. Prokopiou ${ }^{1}$, B. S. Tselentis ${ }^{1} \&$ M. Toanoglou ${ }^{2}$ \\ ${ }^{1}$ Department of Maritime Studies, University of Piraeus, Greece \\ ${ }^{2}$ Euroxenia, Hotel Management and Tourism Consulting, Rhodes, Greece
}

\begin{abstract}
The tourist industry is one of the most important economic activities in Greece. The country receives over 14 million visitors per year. In an era where tourism is one of the fastest growing sectors of the developing countries the competition with existing tourism destinations, such as Greece, will intensify. Greece has enjoyed increasing numbers and revenues from tourism due to the unique environment both natural and man-made making it a high profile tourist destination. However, it is already clear, that the uncontrolled growth of the industry can bring serious environmental and social problems, leading to a decline in the quality of tourist product and services provided. Such negative effects can be controlled and reduced using environmental and tourism indices to estimate the impact of tourism and other businesses on the environment. The purpose of this paper is to apply the principles of coastal environmental management for the Islands in the central Aegean Sea via the carrying capacity assessment methodology in order to develop environmental performance indicators necessary for formulating a novel sustainable development policy proposal for Greek tourism.
\end{abstract}

Keywords: tourism carrying capacity, tourism and environment, tourism development, destinations and environment.

\section{Introduction}

Greece depends heavily on the tourist trade, as tourism is the main economic activity in Greece. The issue for a long term viable development in the Greek islands is to find a creative and hopeful perspective through the carrying capacity assessment indicators. Sustainable development and its quantification procedure 
with specialised measurement instruments, is a vital element for the environmental condition of the Greek destinations; as the tourist product is a blend of ecological, social and economic sub-systems [1, 2]. For that reason an adapted amount of sustainable indicators is selected and a comprehensive methodological guide for their use is created, aiming to reliable measurement of selected parameters that are considered to be important for our region. We believe that using this approach, the local societies, and the stakeholders involved as well, are able to understand the size of the impact on the existing and mainly not renewable resources, in order to proceed with strategic planning and terms of sustainability for their future developmental course. The natural environment is crucial to the attractiveness of almost all travel destinations and recreation areas [3]. Coastal and marine areas are also important recreation resources for local residents as well as the tourists who spend much time with marine activities [4]. Coastal zone management is emerging as a deep concern of governments [5]. Through this study and its results the researcher will try to determine the destination status in order to improve tourism and suggest a plan that will help these islands to become more competitive as tourist destinations. Carrying capacity assessment has become an indispensable tool for formulating policy and strategies in the tourist industry worldwide [6]. Countries and regions with considerable natural and cultural resources look towards tourism when stimulating their sustainable development activities [7]. The contribution of tourism sector to development needs to be clearly explained to allow countries to invest in tourism. Tourism is considered as a development factor [8] as it is probably the best example among human activities in which the linkages between environmental quality and economic prospects are evident [9]. Tourism is a part of a general strategy towards sustainability. There is little agreement on, or evidence of, how to achieve this.

\section{General characteristics}

The Cyclades islands are located at the south part of the Aegean Sea. The Cyclades comprise about 200 islands and the major ones are: Amorgós, Anáfi, Ándros, Antíparos, Dēlos, Íos, Kéa, Kímōlos, Kýthnos, Mēlos, Mykonos, Náxos, Páros, Folégandros, Sérifos, Sífnos, Síkinos, Sýros, Tēnos, and Thēra or Santoríni. Most of the smaller islands are uninhabited [10]. Ermoupolis, on Syros, is the capital town and administrative center of the former prefecture. The islands are peaks of a submerged mountainous terrain, with the exception of two volcanic islands, Milos and Santorini (Thera). The climate is generally dry and mild, but with the exception of Naxos the soil is not very fertile: agricultural production includes wine, fruit, wheat, olive oil, and tobacco. Cooler temperatures are found in higher r. Samos was a prefecture in Greece, consisting of the islands of Samos, Ikaria and the smaller islands of Fournoi Korseon. In 2011 the prefecture was modified and the territory is now covered by the regional units of Samos and Ikaria. Its capital was the town of Vathy, in Samos. The Cycladic islands represent one of the most popular destinations in Greece which is highly dependent on tourism. The purpose of this study is to examine 
the image of the Cycladic and Samos region islands by analysing the interactions between tourism and the environment by examining environmental indices [11]. The population of Cyclades is mainly concentrated in Syros $(19,870)$ Naxos $(18,988)$, Thira $(13,960)$, Paros $(12,853)$, Andros $(1,009)$ Mykonos $(9,320)$ Tinos $(8,574)$, the other islands' population is under 4,000. Samos' population is 33.814 and Ikaria's 8,312. There is only one town with more than 10,000 people, the Capital of Cyclades, Ermoupoli (approximately 15,000).

\section{Tourism development}

Tourism development started mainly in 1980. In 1977 the islands with more than 5 hotels were: Andros (15), Ios (8), Milos (5), Mykonos (17), Naxos (18), Paros (20), Syros (14) and Thira (9) and Tinos (17). In Samos there were at the same time (20) hotels [12]. Massive tourism development in all the Cyclades and Samos islands started after the 90s. Tourism in Cyclades is based in a very unique environment, as the local architectural style, the long sandy beaches, the traditionally built hotels, the nice restaurants with marvelous food, and the hospitable people. Samos region islands are different; there the natural and environment and the unique human environment of Ikaria are the competitive advantages.

\section{Ports and airports}

\subsection{Ports}

In the islands, there are thirty three (33) ports that connect with the other islands and the mainland of Greece.

\subsection{Airports}

Eight airports operate in these islands, and three of them are international: Mykonos, Thira and Samos. The other airports, in Syros, Paros, Naxos, Milos and Ikaria are regional.

\section{Conclusions}

The data presented in Table 1 indicate the major municipalities where this indicator highlights serious coastal pressures on the coastline. From Ermoupoli Town 5226,78 (with tourists per $\mathrm{km}$ of beach), Mykonos $(1837,47$ ) and Folegandros $(1329,23)$ concentrates the most serious pressures at the coasts. Paros, Antiparos and Syros have serious pressures from illegal buildings incidents and marine pollution incidents (tables 5-8). Urban waste management (solid and liquid) on the islands is characterized by lack of efficiency regarding the waste treatment in the cities of Samos/Vathy, the city of Tinos and the city of Naxos. The population of these cities is 6,000 inhabitants for each approximately. That causes serious problems for the tourism industry of these 
places. Moreover it is interesting to note that other smaller settlements do not even have a complete urban waste collection network. (See tables: 9 and 10) The data presented in Table 6 indicates that the passenger arrivals in charter flights in Samos airport have a $19 \%$ decrease as in the airports of the Cyclades islands the arrivals has a smaller decrease. The data presented in Table 9 and 10 indicates the major municipalities where this indicator highlights serious noise pressures at the massive tourism districts as Paros, Thira Ia and Vathy. Regarding fresh water consumption (tables 9 and 10), the demand is increased during the summer months as in all over the island. Some islands they deal with sea waters intrusion incidents into the drinkable water sources. The Greek municipalities do not manage its destination needs with specialized management schemes. Only

Table 1: Beach impact factor and beds per kilometres in the Cyclades islands.

\begin{tabular}{|c|c|c|c|c|c|}
\hline MUNICIPALITY & $\begin{array}{c}\text { TOTAL } \\
\text { TOURISM } \\
\text { CAPACITY } \\
\text { (BEDS) } \\
2010[13] \\
\end{array}$ & $\begin{array}{l}\text { BED } \\
\text { S } \\
/ \mathbf{S Q} \\
\mathbf{K M}\end{array}$ & $\begin{array}{c}\text { HOTEL } \\
\text { BEDS/ } \\
\text { KM OF } \\
\text { BEACH }\end{array}$ & $\begin{array}{l}\text { DESTINATION } \\
\text { MANAGENT } \\
\text { SCHEMES }\end{array}$ & $\begin{array}{c}\text { TOTAL } \\
\text { TOURISM } \\
\text { CAPACITY } \\
\text { (BEDS) } \\
2005[14] \\
\end{array}$ \\
\hline \multicolumn{6}{|c|}{ SYROS ISLAND } \\
\hline ERMOYPOLI TOWN & 2927 & 0,56 & 5226,78 & $\mathrm{NO}$ & 769 \\
\hline POSIDONIA & 2260 & 4,8 & 470,83 & $\mathrm{NO}$ & 763 \\
\hline ANO SYROS & 1281 & 2,8 & 457,5 & $\mathrm{NO}$ & 580 \\
\hline AMORGOS ISLAND & 3395 & 8,5 & 399,41 & $\mathrm{NO}$ & 298 \\
\hline \multicolumn{6}{|c|}{ ANDROS ISLAND } \\
\hline KORTHIO & 197 & 3,5 & 56,285 & $\mathrm{NO}$ & - \\
\hline YDROYSA & 3371 & 15,2 & 221,77 & $\mathrm{NO}$ & 860 \\
\hline ANDROS TOWN & 1307 & 4,2 & 311,19 & $\mathrm{NO}$ & 395 \\
\hline \multicolumn{6}{|c|}{ THIRA ISLAND } \\
\hline THIRA TOWN & 16010 & 18,6 & 860,75 & YES & 8478 \\
\hline IA ISLAND & 1021 & 5,8 & 176,03 & YES & 716 \\
\hline THIRASIA ISL & 44 & 2 & 22 & YES & 38 \\
\hline IOS ISLAND & 4333 & 9,48 & 457,06 & YES & 2129 \\
\hline KEA ISL & 942 & 9,2 & 102,39 & $\mathrm{NO}$ & 295 \\
\hline KITHNOS ISL & 963 & 15 & 64,2 & $\mathrm{NO}$ & 246 \\
\hline MILOS ISLAND & 4393 & 20,5 & 214,29 & YES & 968 \\
\hline MYKONOS ISL & 18926 & 10,3 & 1837,47 & YES & 8881 \\
\hline \multicolumn{6}{|c|}{ NAXOS ISLAND } \\
\hline NAXOS TOWN & 4000 & 18,5 & 170.64 & $\mathrm{NO}$ & 3821 \\
\hline DRYMALIA & 595 & 6,8 & 87,5 & $\mathrm{NO}$ & 96 \\
\hline PAROS ISLAND & 18063 & 22,5 & 802,8 & YES & 6473 \\
\hline SERIFOS ISLAND & 400 & 7,1 & 0 & $\mathrm{NO}$ & 306 \\
\hline SIFNOS ISLAND & 2317 & 4,62 & 501,51 & YES & 832 \\
\hline \multicolumn{6}{|c|}{ TINOS ISLAND } \\
\hline TINOS TOWN & 2495 & 3,2 & 779,68 & $\mathrm{NO}$ & 1794 \\
\hline EXOMBOURG & 773 & 6,3 & 122,69 & NO & 43 \\
\hline PANORMOS & 312 & 1,2 & 260 & $\mathrm{NO}$ & 0 \\
\hline ANAFI ISLAND & 724 & 5,41 & 133,82 & $\mathrm{NO}$ & 0 \\
\hline ANTIPAROS ISL & 2343 & 7,3 & 320,95 & $\mathrm{NO}$ & 343 \\
\hline DONOUSA ISL & 500 & 2 & 250 & $\mathrm{NO}$ & 0 \\
\hline IRACLIA ISL & 230 & 1,5 & 153,33 & $\mathrm{NO}$ & 0 \\
\hline KIMOLOS ISLAND & 228 & 7,5 & 30,4 & $\mathrm{NO}$ & 0 \\
\hline KOUFONISI ISL & 1761 & 2 & 880,5 & $\mathrm{NO}$ & 107 \\
\hline SIKINOS ISLAND & 240 & 1,11 & 216,21 & $\mathrm{NO}$ & 37 \\
\hline SCHINOUSA ISL & 443 & 3,6 & 123,05 & $\mathrm{NO}$ & 58 \\
\hline FOLEGADROS ISL. & 2592 & 1,95 & 1329,23 & $\mathrm{NO}$ & 0 \\
\hline
\end{tabular}


some islands implement specialized know how from scientists or specific companies (Tables 1 and 2). It is obvious that island destinations have to focus on their traditional and authentic characteristics in order to be able to implement a strategy for sustainable development. They need to have an integrated approach to their tourism resources in order to optimize the use of the competitive advantages for their promotion [15]. These approaches can be referred from specialized destination management organizations which will involve all local shareholders. Most Greek municipalities do not manage their destination needs with specialized management schemes. Several islands implement specialized know how with strategic planning from scientists or specialized companies (Tables 1 and 2).

Table 2: Beach impact factor and beds per kilometres in Samos district islands.

\begin{tabular}{|c|c|c|c|c|c|}
\hline MUNICIPALITY & $\begin{array}{l}\text { TOTAL TOURISM } \\
\text { CAPACITY } \\
\text { (BEDS) } 2010\end{array}$ & $\begin{array}{l}\text { BEDS } \\
\text { /SQ KM }\end{array}$ & $\begin{array}{c}\text { HOTEL } \\
\text { BEDS/ KM } \\
\text { OF } \\
\text { BEACH }\end{array}$ & $\begin{array}{l}\text { DESTINATION } \\
\text { SCHEMES } \\
\text { MANAGENT }\end{array}$ & $\begin{array}{c}\text { TOTAL } \\
\text { TOURISM } \\
\text { CAPACITY } \\
\text { (BEDS) } 2005\end{array}$ \\
\hline \multicolumn{6}{|c|}{ SAMOS ISLAND } \\
\hline VATHI & 5034 & 18,95 & 265,64 & NO & 3905 \\
\hline KARLOVASI & 1219 & 4,4 & 277,045 & NO & 1230 \\
\hline $\begin{array}{l}\text { MARATHOKAB } \\
\text { OS } \\
\end{array}$ & 2790 & 8,95 & 311,73 & NO & 930 \\
\hline PYTHAGORIO & 5556 & 9,05 & 613,92 & NO & 3801 \\
\hline \multicolumn{6}{|c|}{ IKARIA ISLAND } \\
\hline AGIOS KYRIKOS & 896 & 5,25 & 170,6667 & NO & 124 \\
\hline EFDILOS & 316 & 2,8 & 112,8571 & NO & 191 \\
\hline RACHES & 932 & 1,55 & 601,2903 & NO & 322 \\
\hline FOURNI ISLAND & 364 & 6,6 & 55,15 & NO & 0 \\
\hline THIMENA ISL & 8 & 1,2 & 6,66 & $\mathrm{NO}$ & 0 \\
\hline
\end{tabular}

Table 3: $\quad$ Tourism capacity history in Samos district islands [16].

\begin{tabular}{|c|c|c|}
\hline & BED CAPACITY IN 2010 & BED CAPACITY IN 2002 \\
\hline SAMOS ISLAND & 14,599 & 16,968 \\
\hline IKARIA ISLAND & 2,144 & 3,024 \\
\hline FOURNI ISLAND & 364 & 351 \\
\hline
\end{tabular}

Table 4: $\quad$ Tourism capacity history in Cyclades islands [17].

\begin{tabular}{|c|c|c|}
\hline & BED CAPACITY IN 2010 & BED CAPACITY IN 2002 \\
\hline MYKONOS & 18,926 & 14,734 \\
\hline THIRA & 17,031 & 22,660 \\
\hline PAROS & 18,063 & 17,804 \\
\hline
\end{tabular}

Table 5: $\quad$ Beach impact factor (illegal buildings incidents per $\mathrm{km}$ of beach).

\begin{tabular}{|c|c|c|c|c|c|c|c|c|c|c|c|}
\hline \multicolumn{9}{|c|}{ BEACH IMPACT FACTOR } \\
\hline ISLAND & 2000 & 2001 & 2002 & 2003 & 2004 & 2005 & 2006 & 2007 & 2008 & 2009 & 2010 \\
\hline IKARIA & 0 & 0 & 0.10 & 0.10 & 0 & 0 & 0.10 & 0 & 0 & 0 & - \\
\hline SAMOS & 0.14 & 0.14 & 0.14 & 0.14 & 0.14 & 0.14 & 0.14 & 0.14 & 0.14 & 0.14 & - \\
\hline
\end{tabular}


Table 6: $\quad$ Beach impact factor (marine pollution incidents per $\mathrm{km}$ of beach).

\begin{tabular}{|c|c|c|c|c|c|c|c|c|c|c|c|}
\hline \multicolumn{1}{|c|}{ BEACH IMPACT FACTOR } \\
\hline ISLAND & 2000 & 2001 & 2002 & 2003 & 2004 & 2005 & 2006 & 2007 & 2008 & 2009 & 2010 \\
\hline IKARIA & 0 & 0 & 0 & 0 & 0 & 0 & 0 & 0 & 0 & 0 & - \\
\hline SAMOS & 0,01 & 0,01 & 0 & 0.07 & 0 & 0 & 0.07 & 0 & 0 & 0.07 & - \\
\hline
\end{tabular}

Table 7: $\quad$ Beach impact factor (illegal buildings incidents per $\mathrm{km}$ of beach).

\begin{tabular}{|c|c|c|c|c|c|c|c|c|c|c|c|}
\hline \multicolumn{10}{|c|}{ BEACH IMPACT FACTOR } \\
\hline ISLAND & 2000 & 2001 & 2002 & 2003 & 2004 & 2005 & 2006 & 2007 & 2008 & 2009 & 2010 \\
\hline SYROS & - & - & - & - & - & 0 & 0 & 0.12 & 0.12 & 0 & 0.12 \\
\hline AMORGOS & 0 & 0 & 0.11 & 0 & 0 & 0.11 & 0 & 0 & 0.11 & - & - \\
\hline ANDROS & - & - & - & - & - & - & - & - & - & - & - \\
\hline THIRA & 0.04 & 0.04 & 0 & 2.33 & 0.04 & 0.12 & 0.12 & - & - & - & - \\
\hline IOS & 0.10 & 0 & 0.10 & 0 & 0.10 & 0 & 0.10 & 0 & 0.10 & 0 & 0 \\
\hline KEA & 0.10 & 0 & 0.10 & 0 & 0 & 0 & 0 & 0 & 0 & 0 & 0.10 \\
\hline KITHNOS & 0.06 & 0 & 0.06 & 0 & 0.06 & 0 & 0.06 & 0 & 0.06 & 0 & 0.06 \\
\hline MILOS & 0 & 0 & 0 & 0 & 0.19 & 0.24 & 0.04 & 0 & - & - & - \\
\hline MYKONOS & - & - & - & - & - & - & - & - & - & - & - \\
\hline NAXOS & 0 & 0 & 0 & 0 & 0 & 0.03 & 0.19 & 0,07 & - & - & - \\
\hline PAROS & 0.16 & 0.16 & 0.16 & 0.16 & 0.16 & 0.16 & 0.16 & 0.16 & 0.16 & - & - \\
\hline SERIFOS & 0 & 0.14 & 0 & 0 & 0 & 0 & 0 & 0.28 & - & - & - \\
\hline SIFNOS & 0.21 & 0.21 & 0.21 & 0.21 & 0.21 & 0.21 & 0.21 & 0.21 & - & - & - \\
\hline TINOS & - & - & - & - & - & - & - & - & - & - & - \\
\hline ANAFI & - & - & - & - & - & - & - & - & - & - & - \\
\hline ANTIPAROS & 0.13 & 0.13 & 0.13 & 0.13 & 0.13 & 0.13 & 0.13 & 0.13 & 0.13 & - & - \\
\hline FOLEGANRO & 0 & 0 & 0 & 0 & 0 & 0 & 0 & 0 & 0.51 & - & - \\
S & & & & & & & & & & & \\
\hline KIMOLOS & 0 & 0 & 0 & 0 & 0 & 0 & 0 & 0 & - & - & - \\
\hline SIKINOS & 0 & 0 & 0 & 0 & 0 & 0 & 0 & 0 & - & - & - \\
\hline
\end{tabular}

In table 8 we present the relation between illegal building incidents with beaches length per $\mathrm{km}$; as in table 9, the marine pollution incidents per $\mathrm{km}$ of beach.

Table 8: Beach impact factor (marine pollution incidents per $\mathrm{km}$ of beach) in Cyclades islands.

\begin{tabular}{|c|c|c|c|c|c|c|c|c|c|c|c|}
\hline \multicolumn{3}{|c|}{ BEACH IMPACT FACTOR } & \multicolumn{9}{|c|}{ (MARINE POLLUTION INCIDENTS PER KM OF BEACH) } \\
\hline ISLAND & 2000 & $\begin{array}{l}2001 \\
{[18]}\end{array}$ & 2002 & 2003 & 2004 & 2005 & 2006 & 2007 & 2008 & 2009 & 2010 \\
\hline SYROS & 0.07 & 0.07 & - & - & - & - & - & - & 0.24 & 0.36 & 1.34 \\
\hline AMORGOS & 0 & 0 & 0 & 0 & 0 & 0 & 0 & 0 & 0 & - & - \\
\hline ANDROS & 0 & 0 & & & & & & & & & \\
\hline THIRA & 0 & 0 & 0 & 0.04 & 0 & 0 & 0 & $\begin{array}{l}0.04 \\
{[19]}\end{array}$ & 0 & 0 & 0 \\
\hline IOS & 0 & 0 & 0 & 0,10 & 0 & 0 & 0 & 0 & - & - & - \\
\hline KEA & 0 & 0.10 & 0 & 0.10 & 0 & 0 & 0 & 0 & 0 & 0.10 & 0.10 \\
\hline KITHNOS & & & & & Insig & ificant & ounts & & & & \\
\hline MILOS & - & - & - & - & - & - & 0.04 & 0 & - & - & - \\
\hline MYKONOS & Insign & icant an & punts & - & - & - & - & - & - & - & - \\
\hline NAXOS & 0 & 0 & 0 & 0 & 0 & \begin{tabular}{|l|}
0.03 \\
\end{tabular} & 0.07 & 0.03 & - & - & - \\
\hline PAROS & 0.05 & 0.05 & 0.05 & 0.05 & 0.05 & 0.05 & 0.05 & 0.05 & - & - & - \\
\hline SERIFOS & 0 & 0 & 0 & 0 & 0 & 0 & 0 & 0 & - & - & - \\
\hline SIFNOS & 0 & 0 & 0 & 0 & 0 & 0 & 0 & 0 & - & - & - \\
\hline TINOS & - & - & - & - & - & - & - & - & - & - & - \\
\hline ANAFI & 0 & 0 & - & - & - & - & - & - & - & - & - \\
\hline ANTIPAROS & 0 & 0 & 0 & 0 & 0 & 0 & 0 & 0 & 0 & - & - \\
\hline FOLEGANDROS & 0 & 0 & 0 & 0 & 0 & 0 & 0 & 0 & 0 & - & - \\
\hline KIMOLOS & - & - & - & - & - & - & - & - & - & - & - \\
\hline SIKINOS & 0 & 0 & 0 & 0 & \begin{tabular}{|l|l} 
\\
\end{tabular} & 0 & 0 & 0 & 0 & 0 & 0 \\
\hline
\end{tabular}


Table 9: Waste management and noise nuisance in the Cyclades islands.

\begin{tabular}{|c|c|c|c|c|}
\hline & $\begin{array}{l}\text { URBAN WASTE } \\
\text { TREATED } \\
\text { COVERAGE \% }\end{array}$ & $\begin{array}{c}\text { GARBAGE } \\
\text { MANAGEMENT }\end{array}$ & $\begin{array}{l}\text { SEA WATER } \\
\text { INTRUSION } \\
\text { INCIDENTS }\end{array}$ & $\begin{array}{c}\text { NOISE NUISANCE } \\
\text { MAXIMUM AMOUNT: } \\
5 \text { POINTS }\end{array}$ \\
\hline ERMOYPOLI TOWN & $100 \%$ & NLS & $\mathrm{NO}$ & 1 \\
\hline POSIDONIA & $\mathrm{UC}$ & NLS & YES & 1 \\
\hline ANO SYROS & $20 \%$ & NLS & NO & 0 \\
\hline AMORGOS ISL. & $\mathrm{UC}$ & IS & $\mathrm{NO}$ & 0 \\
\hline KORTHIO & $\mathrm{UC}$ & LS & $\mathrm{NO}$ & 0 \\
\hline YDROYSA & $\mathrm{UC}$ & LS & YES & 0 \\
\hline ANDROS TOWN & $\mathrm{UC}$ & LS & NO & 0 \\
\hline THIRA TOWN & $95 \%$ & LS & YES & 2 \\
\hline IA & $90 \%$ & LS & NO & 2 \\
\hline THIRASIA ISLAND & 0 & LS & $\mathrm{NO}$ & 0 \\
\hline IOS ISLAND & $90 \%$ & NLS & YES & 1 \\
\hline KEA ISLAND & 0 & IS & YES & 1 \\
\hline KITHNOS ISLAND & 0 & NLS & $\mathrm{NO}$ & 1 \\
\hline MILOS ISLAND & 0 & IS & $\mathrm{NO}$ & 1 \\
\hline MYKONOS ISLAND & $98 \%$ & NLS & $\mathrm{NO}$ & 1 \\
\hline NAXOS TOWN & 0 & IS & $\mathrm{NO}$ & 2 \\
\hline DRYMALIA & $60 \%$ & IS & $\mathrm{NO}$ & 0 \\
\hline PAROS ISLAND & $70 \%$ & NLS & YES & 2 \\
\hline SERIFOS ISLAND & $70 \%$ & LS & $\mathrm{NO}$ & 0 \\
\hline SIFNOS ISLAND & $12 \%$ & IS & NO & 0 \\
\hline TINOS TOWN & 0 & IS & NO & 1 \\
\hline EXOMBOURG & 0 & IS & YES & 0 \\
\hline PANORMOS & 0 & IS & NO & 0 \\
\hline ANAFI ISLAND & 0 & LS & NO & 1 \\
\hline ANTIPAROS ISLAND & 0 & IS & YES & 1 \\
\hline DONOUSA ISLAND & 0 & IS & $\mathrm{NO}$ & 0 \\
\hline IRACLIA ISLAND & 0 & IS & $\mathrm{NO}$ & 0 \\
\hline KIMOLOS ISLAND & 0 & IS & $\mathrm{NO}$ & 0 \\
\hline KOUFONISI ISLAND & $100 \%$ & IS & $\mathrm{NO}$ & 1 \\
\hline SIKINOS ISLAND & 0 & IS & $\mathrm{NO}$ & 0 \\
\hline SCHINOUSA ISLAND & 0 & IS & $\mathrm{NO}$ & 0 \\
\hline FOLEGADROS ISL & 0 & NLS & YES & 1 \\
\hline
\end{tabular}

Table 10: Waste management and noise nuisance in Samos district islands. Maximum amount for each municipality (noise nuisance).

\begin{tabular}{|c|c|c|c|c|}
\hline & $\begin{array}{c}\text { URBAN WASTE } \\
\text { TREATED } \\
\text { COVERAGE } \%\end{array}$ & $\begin{array}{c}\text { GARBAGE } \\
\text { MANAGEMENT } \\
\text { (ABBREVIATIONS } \\
\text { IN TABLE 9) }\end{array}$ & $\begin{array}{c}\text { SEA } \\
\text { WATER } \\
\text { INTRUSION } \\
\text { INCIDENTS }\end{array}$ & $\begin{array}{c}\text { NOISE } \\
\text { NUISANCE } \\
\text { MAXIMUM } \\
\text { AMOUNT: } \\
\text { 5 POINTS }\end{array}$ \\
\hline VATHI & $11 \%$ & NLS & NO & 3 \\
\hline KARLOVASI & $62 \%$ & DE & NO & 1 \\
\hline MARATHOKABOS & $8 \%$ & DE & NO & 0 \\
\hline PYTHAGORIO & $38 \%$ & NLS & NO & 1 \\
\hline AGIOS KYRIKOS & 0 & NLS & NO & 0 \\
\hline EFDILOS & $25 \%$ & NLS & NO & 0 \\
\hline RACHES & 0 & NLS & NO & 0 \\
\hline FOURNI ISLAND & 1 & UC & NO & 1 \\
\hline THIMENA ISLAND & 0 & IS & NO & 0 \\
\hline
\end{tabular}

LS: Landfill sites for municipal waste, RLS: Landfill sites for residual municipal waste (after recycling), NLS: Landfill sites for municipal waste non attending to 1999/31/EU, IS: Illegal sites for municipal waste, DE: Destroyed. 
Table 11: $\quad$ Total arrivals in 2010 and 2007.

\begin{tabular}{|c|c|c|c|c|c|c|}
\hline \multirow[t]{2}{*}{ ISLANDS } & \multicolumn{2}{|c|}{$\begin{array}{c}\text { PASSENGER } \\
\text { ARRIVALS IN PORTS }\end{array}$} & \multicolumn{2}{|c|}{$\begin{array}{c}\text { PASSENGER } \\
\text { ARRIVALS FROM } \\
\text { DOMESTIC } \\
\text { FLIGHTS } \\
\end{array}$} & \multicolumn{2}{|c|}{$\begin{array}{c}\text { PASSENGER ARRIVALS } \\
\text { FROM INTERNATIONAL } \\
\text { FLIGHTS }\end{array}$} \\
\hline & 2007 & 2010 & 2007 & 2010 & 2007 & 2010 \\
\hline SYROS & 337,450 & 266,547 & 3,612 & 6,796 & 0 & 0 \\
\hline AMORGOS & 6,2955 & 58,384 & - & - & - & - \\
\hline ANDROS & 348,422 & 270,571 & - & - & - & - \\
\hline THIRA & 546,625 & 527,936 & 167,242 & 161,553 & 192,042 & 181,746 \\
\hline IOS & 73,696 & 103,595 & - & - & - & - \\
\hline KEA & 165,864 & 145,516 & - & - & - & - \\
\hline KITHNOS & 76,423 & 66,727 & - & - & - & - \\
\hline MILOS & 135,519 & 77,473 & 18,121 & 18,049 & 0 & 0 \\
\hline MYKONOS & 596,212 & 439,504 & 106,690 & 100,219 & 104,901 & 111,136 \\
\hline NAXOS & 380,551 & 330,020 & 10,908 & 10,112 & 2,167 & 766 \\
\hline PAROS & 590,742 & 501,879 & 21,079 & 20,532 & 0 & 0 \\
\hline SERIFOS & 69,480 & 61,132 & - & - & - & - \\
\hline SIFNOS & 25,725 & 99,530 & - & - & - & - \\
\hline TINOS & 627,347 & 413,481 & - & - & - & - \\
\hline ANAFI & 8798 & 11,399 & - & - & - & - \\
\hline DONOUSA & 7,539 & 7.913 & - & - & - & - \\
\hline IRACLIA & 6,094 & 6,782 & - & - & - & - \\
\hline KIMOLOS & 25,725 & 8,893 & - & - & - & - \\
\hline KOUFONISI & 25,930 & 31,580 & - & - & - & - \\
\hline SHINOUSA & 8,512 & 9,431 & - & - & - & - \\
\hline SIKINOS & 8,925 & 32,739 & - & - & - & - \\
\hline SAMOS & 138,164 & 101,551 & 105,823 & 94,025 & 132.228 & 108,882 \\
\hline IKARIA & 94,464 & 76,804 & 12,714 & 12,419 & 0 & 0 \\
\hline FOURNI & 14,989 & 14,591 & - & - & - & - \\
\hline THIMENA & 624 & 2348 & - & - & & - \\
\hline
\end{tabular}

\section{Criteria scoring evaluation}

In table 12 we present the results from the above indices regarding the highest rates of environmental impacts, combined with the level of tourist arrivals and the destination management.

Samos Island: has experienced the greatest reduction in tourist arrivals, in hotel capacity as it does not have tourism destination management. That means that Samos confronts serious destination problems.

Vathy: has serious noise nuisance problems.

Ermoupoli: has serious coastal pressures on the coastline (marine pollution incidents as tourists per km of beach).

Thira: has the highest amount of illegal buildings and other illegal construction on the coasts.

Andros, Tinos, Anafi and Mykonos port authorities did not answer the questionnaires for its coasts.

Vathy, Tinos, and Naxos towns with more than 7 thousand inhabitants each do not have any urban waste treatment plants. 
Table 12: Criteria scoring.

\begin{tabular}{|c|c|c|c|c|}
\hline $\begin{array}{l}\text { HOTEL BEDS/ KM OF } \\
\text { BEACH }\end{array}$ & $\begin{array}{c}5226,78 \\
\text { ERMOUPOLI }\end{array}$ & $\begin{array}{c}1329,23 \\
\text { FOLEGANDROS }\end{array}$ & $\begin{array}{c}1837,47 \\
\text { MYKONOS }\end{array}$ & $\begin{array}{l}860,75 \\
\text { THIRA } \\
\text { TOWN }\end{array}$ \\
\hline $\begin{array}{c}\text { DESTINATION } \\
\text { MANAGENT SCHEMES } \\
\end{array}$ & \multicolumn{4}{|c|}{33 MUNICIPALITIES WITHOUT } \\
\hline $\begin{array}{l}\text { BEACH IMPACT FACTOR } \\
\text { /ILLEGAL BUILDINGS } \\
\text { PER KM OF BEACH }\end{array}$ & $\begin{array}{l}2.33 \text { THIRA } \\
\text { ISLAND }\end{array}$ & 0.21 SIFNOS & $0.19 \mathrm{NAXOS}$ & 0.16 PAROS \\
\hline $\begin{array}{c}\text { BEACH IMPACT FACTOR } \\
\text { / MARINE POLLUTION } \\
\text { INCIDENTS PER KM OF } \\
\text { BEACH } \\
\end{array}$ & 1.34 SYROS & & & \\
\hline URBAN WASTE TREATED & \multicolumn{4}{|c|}{16 MUNICIPALITIES WITH $0 \%$ COVER } \\
\hline GARBAGE & 12 MUNICIPA & IIES WITH ILLEG & TE FOR MUN & ALI WASTE \\
\hline NOISE NUISANSE & 3 VATHY & 2 THIRA TOWN & 2 IA & 2 PAROS \\
\hline $\begin{array}{c}\text { FERRIES PASSENGER } \\
\text { ARRIVALS REDUCTION }\end{array}$ & $\begin{array}{c}\text { TINOS } \\
35 \% \\
\end{array}$ & $\begin{array}{c}\text { SAMOS } \\
28 \% \\
\end{array}$ & $\begin{array}{c}\text { MYKONOS } \\
27 \% \\
\end{array}$ & $\begin{array}{c}\text { ANDROS } \\
22 \% \\
\end{array}$ \\
\hline $\begin{array}{c}\text { AIRPORTS } \\
\text { INTERNATIONAL } \\
\text { ARRIVALS REDUCTION }\end{array}$ & $\begin{array}{l}\text { SAMOS } \\
\text { ISLAND } \\
-19 \% \\
\end{array}$ & THIRA - $6 \%$ & & \\
\hline $\begin{array}{c}\text { AIPORTS DOMESTIC } \\
\text { ARRIVALS REDUCTION } \\
\end{array}$ & $\begin{array}{c}\text { SAMOS } \\
11 \% \\
\end{array}$ & $\begin{array}{c}\text { MYKONOS } \\
6 \% \\
\end{array}$ & PAROS $5 \%$ & THIRA $4 \%$ \\
\hline $\begin{array}{l}\text { TOURISM CAPACITY } \\
\text { REDUCTION }\end{array}$ & $\begin{array}{l}\text { SAMOS } \\
14 \%\end{array}$ & & & \\
\hline $\begin{array}{c}\text { SEA WATER INTRUSION } \\
\text { INCIDENTS IN } \\
\text { DRINKABLE WATER } \\
\text { SOURCES } \\
\end{array}$ & \multicolumn{4}{|c|}{9 MUNICIPALITIES } \\
\hline
\end{tabular}

\section{Proposals}

Sustainable tourism must be developed in the islands, as well as in all Greek destinations [20]. The conclusion is that the saturated areas can progress towards developing quality and alternative tourist services. Areas that are not yet massively developed should not necessarily develop to the same extent as the major tourist attraction areas, but should, at this stage of development, plan ahead in order that policy development and implementation, lead to a truly competitive and environmentally sound business [21]. The target is a combination of typical and alternative tourist models related to the local community needs and the unique environment. Despite their relatively small area, all the islands have an exceptionally rich nature, with a greatly varying landscape, natural habitats, flora, fauna and vegetation [22]. Tourist development must target using a combination of the classical tourist models and alternative tourist activities, as the environments, both physical and human are unique: 1). Ecotourism: Footpaths, winding roads and climbing, 2). Diving, 3). Archaeological paths, 4). Museums, 5). Traditional villages 6). Cultural tourism related with Orthodox Religion traditional events and 7). Tourism as cultural attractions in order to extend the tourist season [23]. Tourism industry must be extended by supporting winter time charter flights [24]. Completion of urban waste treatment plants and network, appropriate garbage handling (urban garbage treatment - olive oil press residuals management), restriction of illegal 
buildings at cities and coasts, control of hotels' urban waste management systems.

\section{The project of the Tourism Organization of Samos Prefecture}

Samos Prefecture Organization of Tourism was founded in 1996. Since January 2009 it has been operated by a new management group. The president of the organization was the Prefect of Samos Dr E. Carlas and Manager of the Organization is Mr. M. Toanoglou a hotel owner and destination consultant from Rhodes. The assistant manager is D.G. Prokopiou, a Phd candidate in tourism from the University of Piraeus. The aim of OTONAS was to identify the competitive advantages of the region and clarify the current status concerning tourism and hospitality on the 3 island sub destinations (Samos-Ikaria - Fournoi).

Additionally many efforts were focused to the participation of the main stakeholders. The aim was for everybody to understand that tourism should be the priority. As tourism is the main economic factor. The main activities of the 2 year project of OTONAS under the mentioned management scheme were: 1). Identifying the potential resources that could improve the competitiveness of the region and improve the image to the tourism markets. 2). Selecting 2-3 new foreign tourism markets and implementing strategic promotion to open the destination in those new markets (e.g., Russia, France, etc.). 3). Improving the image and the promotion in the existing tourism markets with specific actions (roadshows, workshops, join activities with tour operators, better participation at tourism exhibitions, etc.) 4). Involving the destination in wider (EU and International) programs that could lead the islands to a higher level of organization and competitiveness. It is true that the main difficulty of that program was the lack of common understanding and perception of the involved shareholders. In many cases political opinions of individuals or authorities were blocking decisions of common benefit. The policy of the management team was many times consumed on efforts that were focused in "training" and "education" of the involved parties in order to improve their tourism mentality and the whole destination awareness. Now as yet, Samos does not have a management scheme and tourism is not governed by a specific establishment organization or from a contract with a destination management company.

\section{Acknowledgements}

We would like to thank for their help and collaboration: Dr Em. Carlas, A. Manousou Attorney at Law, D. Tselou Economist, K. Kostavaras, A. Kargadou, Lieutenant J. Vamvakousis and Sublieutenant M. Daleziou, Syros Port Authority and all the Hellenic Coast Guard port authorities and municipalities of the islands of the study. 


\section{References}

[1] Briassouls H., Policy and Practice, Sustainable Development and its indicators: Though a (planner's) Glass Darkly, Journal of Environmental planning and Management vol. 44(3), 409-427, (2001).

[2] Zannou V, 'Guide of Socio-Economic Studies for the Integrated Management of the Water Environment' (1999).

[3] Farrell B., Runyan D., Ecology and Tourism, Annals of Tourism Research, (1991).

[4] Needham M., Szuster B., Situational influences on normative evaluations of coastal tourism and recreation management strategies in Hawaii, Tourism Management 32, 732-740, (2011).

[5] Charlier R., De Meyer C, Tourism and the coastal zone: The case of Belgium, Ocean and Coastal Management 18, 231-240, (1992).

[6] Prokopiou D.G., Tselentis B.S., Bousbouras D. and Toanoglou M. "Carrying capacity assessment in tourism: The case of Dodecanese archipelago" The Ravage of the planet, First International Conference on the Management of Natural Resources, Sustainable Development and Ecological Hazards, Dec. 2006 Bariloche, Argentina Wessex Institute of Technology UK - University of Siena.

[7] Jarmozy U., Sustainable tourism development: ingenuity in marketing strategy, Sustainable Tourism III International Conference, Malta 2008, Wessex institute of Technology UK- The Compulence University, Spain.

[8] Ntibanyurwa A., Tourism as a factor of development, Sustainable Tourism II International Conference, Bologna 2006,Wessex institute of Technology UK - The Compulence University, Spain.

[9] Priestley G.K., Edwards J.A. and Coccossis H., Sustainable TourismEuropoean Experiences, Cab International 1996 page ix.

[10] www.wikipedia.com

[11] Prokopiou, D.G. Tselentis B.S. Bousbouras D and Toanoglou M. "Sustainability indicators and their role to destinations' Strategic development" Sustainable tourism as a factor of local development Conference, Monza, Italy 7-9/11/2008.

[12] Greece Hotels 1977 Le chamber des Hotels de Grece, Athens.

[13] Actual data collected from Hotel Unions and Municipalities.

[14] Official data from Hellenic Statistics Authority.

[15] Blain C., Levy S., Brent-Richi J.R. Destination Branding: Insights and Practices from destination Management Organizations, Journal of Travel Research, Vol 43, May 2005, Sage Publications.

[16] Tourism organization of Samos Prefecture, 2010.

[17] Papanikoli E., Kretsis M., Study for Tourism in Mykonos, (in Greek) Mytilini 2004.

[18] Tselentis BS - Prokopiou DG, "Environmental Management of Port Oil Terminals," International Conference Rhodes 2002, Wessex Institute.

[19] Shipwreck of a cruise ship in Gulf is Fira (very serious incident). 
[20] Prokopiou D.G. and Tselentis B.S. 'Proposals for sustainable development and environmental protection for the island of Rhodes', Rhodes 2003, Publication: Rodiaki 27 -5-04 (in Greek).

[21] Prokopiou D.G., Tselentis B.S., Bousbouras D. and Toanoglou M. Carrying capacity assessment for the Greek islands of Kalymnos, Kos and Rhodes, Sustainable Tourism II International Conference, Bologna 2006, Wessex institute of Technology UK - The Compulence University, Spain - 'Rodiaki' news 2006.

[22] Prokopiou DG, Tselentis BS, Bousbouras D. and Toanoglou M. "Environmental impacts caused by the tourist industry in Elafonisos Island and the Neapoli district, Greece" ECOSUD 2007, Sixth International Conference on Ecosystems and Sustainable Development, Organised by: Wessex Institute of Technology, UK -The University of Coimbra, 4-6 September 2007 Coimbra, Portugal.

[23] Prokopiou DG, MBA Dissertation: "Regional Investment Study for Kos and Rhodes islands", University of Piraeus, 2005.

[24] Tselentis B.S., Prokopiou D.G., Tourism carrying capacity assessment and the environment: the case of Crete. International Conference on Ecosystems and Sustainable Development, Alicante 2011. 